difficulty in foreign stations in securing volunteers for inoculation ; for instance, about 93 per cent. of the British garrison in India have been protected by inoculation, and typhoid fever, which used to cost us from 300-600 deaths annually, was last year responsible for less than 20 deaths. 4. Inoculation was made compulsory in the American army in 1911 and has practically abolished the disease; in 1913 there were only three cases and no deaths in the entire army of over 90,000 men.

The organisation for the preparation and distribution of the vaccine should be equal to all the demands made upon it. In view of the possibility of such an emergency as has now arisen, arrangements were made some years ago at the Royal Army Medical College to prepare and maintain a very large reserve of the vaccine, and from this reserve we have been able to issue, since mobilisation, more than 170,000 doses for the use of the troops. The vaccine department at the Royal Army Medical College, under Major D. Harvey, will continue for the present the preparation of the vaccine, and we are also fortunate in receiving most generous and valuable assistance from the staff of the Lister Institute and from Sir Almroth Wright. The ideal of universal protection is, I fear, too much to hope for, but with the support of the profession, on which I feel sure we may count, we may hope to save many valuable lives and to minimise one of the gravest causes of depletion of the fighting force.-I am, Sir, yours faithfully,

War Office, August 16th, 1914.

W. B. LEISHMAN, Colonel, R.A.M.C.

\section{THE PRESCRIBING OF NARCOTIC DRUGS.} To the Editor of THE LANCET.

SIR,-The remarks upon the prescribing of narcotic drugs in your leading article in THE LANCET of August 15th are well inspired. It is more than time that new regulations were introduced in this country to check the indiscriminate distribution of drugs to the public. Personally I maintain that no drug of any kind should be dispensed save on production of a prescription written and signed by a medical man. The incalculable harm occasioned by the use of drugs unauthorised is only known to those who, like myself, have made somewhat extensive observations on the subject. Within the last decade I have come across people who were using, without medical advice, the following drugs amongst others: phenacetin, digitalis, nux vomica, pulv. ipecacuanhæ, co., calomel, thyroideum siccum, aspirin, salicylate of soda, bromides, iodides, tr. chloroformi et morphinæ co., tinct. opii, preparations of squills (commonly given to infants), sulphonal, veronal, chloral hydrate, and tinct. belladonnæ. The ease with which these drugs can be obtained by the man in the street is a national scandal calling for instant check.

In writing prescriptions even for quite harmless remedies $I$ am in the habit of appending my signature, and not merely my initials, as the latter do not necessarily convey to the pharmacist the knowledge that a duly qualified person has written the prescription. This practice I teach to students, and also impress upon them the necessity for writing across any prescription that should not be repeated the necessary words to that effect. Many to whom I have spoken on the subject have scoffed at my " unnecessary carefulness," but the time has come when probably my teaching will receive recognition in high quarters, and my opponents will be forced to fall into line with a practice which I have adopted ever since I was in a position to prescribe for the sick. Surely too great care cannot be taken for the safety of the public, and your valuable leading article on the subject will help to rouse the profession from their lethargy regarding the need for care in the writing of their prescriptions in future.

I am, Sir, yours faithfully,

James Burnet, M.A., M.D., M.R.C.P. Edin.

Edinburgh, August 18th, 1914.

\section{ORAL SEPSIS AND THE EXTRACTION OF TEETH. \\ To the Editor of THE LANCET.}

SIR,-It was not my intention to raise a controversy, but to call in question only a dangerous line of practice that seemed to me to be developing, so I do not think it necessary, or even desirable, to make any detailed reply either to Mr. J. G. Turner or Mr. F. St. J. Steadman, who alone have criticised my letter to you adversely. But I would like to say this to make my exact position clear - that each of these gentlemen seems to me to hold that pyorrhoea is a common disease, an insidious one, easily overlooked, yet associated with definite changes in the bones, and that it leads to septic intoxication. If what $I$ in $m y$ ignorance call gingivitis they call pyorrhcea alveolaris, so be it. My essential point is, however, is it the cause of septic absorption, and is it worthy of the extraction of the teeth in bulk?

Now I will admit Mr. Steadman's impeachment that I have no pretension to being an expert in dentistry, but I am already too familiar with the term "pockets" which both he and Mr. Turner speak of, and I am always on the look-out for them in my poor non-expert way, and I should suppose that anything worthy of such a description should contain pus, exuding under pressure such as the eye could see. Yet neither to my sight nor to pressure of the finger along the gums have I been able to detect this in a considerable number of cases. I certainly "have not taken the trouble to wrap a wisp of cotton-wool round a very thin bristle, and gently passed it round each tooth to find them," nor have I "warmed the bristle after withdrawing it, and held it under my nose." I am quite sure that the teeth of very few of us would stand that test; indeed, if that be my critic's criterion of pyorrhœaalveolaris I can only suppose that he has already immolated all his own ivories and been exceptionally happy in the set of "made-at-homes" with which he has supplied himself. To one not an expert it seems more reasonable to hold that so long as teeth are good, firm in their sockets, free from pain, and serviceable for easy mastication, there is something to be said on the side of keeping our own rather than taking to others that we know not of. We seem to be far too ready to jump from the latest hypothesis of oral sepsis to the proof thereof. The changes said to occur in the bone in these cases, for example, have they been clearly distinguished from those that must exist in company with retrocession of the gum?-an exceedingly common condition that carries with it usually no sign of sepsis.

Again, what adequate evidence is there to prove that "the ulceration of pyorrhcea not found patent on the edges of the gums, but hidden on the toothward side of the gum flaps, that help to form the pockets of pyorrhœa"-if it exist-" frequently 
gives rise to gross joint lesions, and many other maladies too numerous to mention." I do not deny it; all I ask for is evidence. Effects from certain causes are too readily assumed. When first the oral sepsis explanation of pernicious anæmia was announced there was the usual hue and cry. How far has it been substantiated? I have seen many a case of pernicious anæmia since then. I cannot record one-I had seen one such before-where attention to the teeth has had any certain effect upon the disease, and it can be but seldom that the suggestion can have helped to its cure. I am, Sir, yours faithfully,

August 16th, 1914. JAMES F. GOODHART.

\section{To the Editor of THE LANCET.}

SrR,-As a contribution to the discussion relating to this subject now raised in your columns I should like to mention a case seen by me four years ago. The patient, a lady of a lifetime of imaginary disorders, drew my attention to the fact that she had undergone extraction of her entire set of teeth since I had seen her a year previously. On further inquiry she informed me that her dentist, whom she had consulted for some trivial dental trouble, had informed her she was suffering from "Rigg's disease," and that it would be necessary to extract all her teeth. Her imaginary ailment at the moment was colitis; this she was assured was caused by "Rigg's disease," and in the event, and with the sanction of her medical attendant, she was rendered edentulous. She assured me with pride that her teeth when drawn were perfectly sound, and I have no doubt they were.

The impression made on my mind at the time was that $I$ had never in a lengthy and varied experience seen so cruel and unwarrantable a pro. cedure under the guise of treatment. In the name of a disease which was not present she was subjected to this outrage for the relief of a condition which was non-existent. It is to be hoped now that the subject has been called attention to under the authority of Sir James Goodhart's name that this form of malpraxis may cease.

I am, Sir, yours faithfully,

C. KNox Bond, M.R.C.S.Eng., L.R.C.P.Lond. Builth Wells, August 17th, 1914.

\section{TUBERCULOSIS SOCIETY.}

\section{To the Editor of THE LANCET.}

SIR,-Recent letters received by the honorary secretary and myself show that, in spite of some recent correspondence in your columns on this matter, the aims and objects of this society are not sufficiently known to those groups of practitioners from which the membership of the society is drawn. The formation of the Tuberculosis Society was a natural outcome of the development of the Tuber. culosis Service. In 1910 a small clinical society was formed in London whose members for the most part were medical officers to tuberculosis dispensaries and to sanatoriums. The rapid development of the Tuberculosis Service throughout the country led to the formation of a larger society in 1912.

The work of the Tuberculosis Service is so special in itself that the need of a separate society was at once apparent. The aim of the Tuberculosis Society is to provide a medium for the exchange of experience, for conference, for discussion of points arising in the course of the work both clinical and administrative, and to safeguard and protect the interests of tuberculosis officers and of the Tuberculosis Service. Meetings are held in London on the third Saturday of every month from October to June. At these meetings papers on various aspects of the work are read and discussed, being published later in the quarterly Transactions of the society. Three times a year the society travels to different provincial centres, where meetings are held for the benefit of members in the provinces.

At the annual meeting the office-bearers and committee for the year are elected. The honorary Presidents of the society are Sir Robert W. Philip and Sir William Osler, and the honorary Vice-Presidents include Dr. Arthur Newsholme, Dr. J. Edward Squire, and Dr. Vincent Bowditch, of Boston. At a recent meeting of the society held in the Town Hall of Leeds, and attended by over 50 tuberculosis officers from various parts of the country, it was recom. mended that the annual meeting of the society be held at the same time and place as the annual conference of the National Association for the Prevention of Consumption. The subscription is $10 \mathrm{~s}$. entrance fee and $5 \mathrm{~s}$. per annum.

The work of this society during the past year may be briefly indicated. Papers were read and discussed on such varied aspects of tuber. culosis as the $\mathrm{X}$ Ray Diagnosis of Early Pulmonary Tuberculosis, the Surgery of Pulmonary Tuberculosis, the Present Position of Tuberculin Treatment, the Growth of Legislation Affecting Tuberculosis, a Case of Acute Miliary Tuberculosis with Macroscopic and Microscopic Specimens, and the Future of the Tuberculosis Service. During the past year matters affecting the status and position of the Tuberculosis Service have occupied a considerable portion of the time of the society, whose present membership includes over 100 tuberculosis officers. As a result of recent meetings attended by tuberculosis officers from all parts of England, a memorial in the following terms was drawn up, approved, and has been submitted to the Chancellor of the Exchequer and to the President of the Local Government Board.

\section{Memorial.}

Your Memorialists represent the Tuberculosis Society. The Tuberculosis Society is a body including in its ranks Tuberculosis Officers appointed to County Councils, County Borough Councils, and the Metropolitan Borough Councils in England, and to County Councils and Town Councils in Scotland, and also Medical Officers to sanatoriums, farm colonies, and hospitals for advanced cases. The newly constituted Tuberculosis Service consists of medical men engaged in the diagnosis, treatment, and prevention of tuberculosis under public bodies.

For the proper discharge of the duties, which recent legislation in relation to tuberculosis demands, special training and experience are essential. An endorsement of the view that Tuberculosis Officers should be specialists in the work is to be found in the recommendations of the Departmental Committee on Tuberculosis, 1913.

The Local Government Boards have endorsed generally the recommendations of the Departmental Committee and proceeded on the view that these officers are to be regarded as an independent service so far as their clinical duties are concerned, and shall be responsible for the management of institutions to which they are attached.

It seems to your memorialists most important that from the outset a satisfactory status and security of tenure should be insured. Without this it will be difficult to induce the best class of men to enter a service for which years of special training are required. Your memorialists consider it necessary to emphasise the fact that the tuberculosis service is mainly clinical in its duties, and does not therefore naturally lead on to transference to the ordinary administrative posts in the Public Health Service. To regard the Tuberculosis Service as a subsidiary branch of the Public Health Service would be to discourage special training in -a 